\title{
Blast from explosive evaporation of carbon dioxide: experiment, modeling and physics
}

\author{
M. M. van der Voort • A. C. van den Berg • \\ D. J. E. M. Roekaerts · M. Xie • P. C. J. de Bruijn
}

Received: 25 January 2011 / Revised: 18 October 2011 / Accepted: 9 January 2012 / Published online: 21 February 2012

(C) The Author(s) 2012. This article is published with open access at Springerlink.com

\begin{abstract}
Explosive evaporation of a superheated liquid is a relevant hazard in the process industry. A vessel rupture during storage, transport or handling may lead to devastating blast effects. In order to assess the risk associated with this hazard or to design protective measures, an accurate prediction model for the blast generated after vessel rupture is needed. For this reason a fundamental understanding of the effects of a boiling liquid expanding vapor explosion (BLEVE) is essential. In this paper, we report on a number of well-defined BLEVE experiments with 40-1 liquid $\mathrm{CO}_{2}$ bottles. The existing inertia-limited BLEVE model has been validated by its application to these experiments. Good qualitative agreement between model and experiment was found, while quantitatively the results provide a safe estimate. Possible model improvements taking into account the finite rate of evaporation are described. These comprise phenomena such as bubble nucleation and growth rate, and the two-phase flow regime. Suggestions for improved experiments are given as well.
\end{abstract}

Keywords BLEVE - Explosive evaporation - Blast . Nucleation · Two-phase flow

Communicated by V.K. Kedrinskii.

M. M. van der Voort $(\varangle)$ · A. C. van den Berg · P. C. J. de Bruijn TNO, P.O. Box 45, Lange Kleiweg 137,

2280 AA Rijswijk, The Netherlands

e-mail: martijn.vandervoort@tno.nl

D. J. E. M. Roekaerts · M. Xie

Department of Multi-Scale Physics,

Delft University of Technology, P.O. Box 5,

2600 AA Delft, The Netherlands

\section{Introduction}

Explosive evaporation of superheated liquids is a hazard faced in many sectors of the process industry. The burst of a pressure vessel containing liquefied gases such as, for instance LPG, ammonia or carbon dioxide is a scenario considered in many industrial hazard evaluations. We use the acronym BLEVE (boiling liquid expanding vapor explosion) to denote the creation of a blast wave by the rapid evaporation process. Note that the possible chemical explosion following the mixing of the released vapor with air is left out of consideration.

Traditionally, the strength of a BLEVE blast is obtained from an energy estimate [1-3]. Underlying these energy estimates is an assumption that the energy release in the source area (the evaporation rate) is high. A loss factor representing how much of the energy is used for the acceleration of projectiles rather than for the creation of a blast wave can be taken into account. Such estimates represent idealized situations, with spherical symmetry, or hemi-spherical symmetry. To be more general and flexible, the geometry of the specific system and the equations for phase change and for blast wave generation have to be taken into account.

Under normal conditions, liquid and vapor in the vessel are in thermodynamic equilibrium with each other. When the vessel ruptures, the pressure suddenly drops to ambient pressure. In equilibrium at lower pressure a larger fraction is vapor and the system will evolve in that direction by evaporation. The quick phase change from liquid to vapor causes a large volume increase. One cubic meter of liquid carbon dioxide, for instance, gives rise to more than hundred cubic meters vapor under atmospheric conditions. Before the evaporation starts, the superheated liquid is in a metastable state. At this moment the temperature is above the boiling temperature at ambient pressure. Depending on the evaporation rate, 
the volume of the system will increase. Ambient air will be pushed aside, with a blast wave as possible consequence.

At small overheating the evaporation will only start at evaporation nuclei at walls and at impurities or ions in the liquid. The evaporation rate is then far too low for the development of any significant blast effect. Reid [4,5] observed, however, that explosive evaporation occurs when the liquid temperature is above some temperature level, namely the superheat limit temperature (also called homogeneous nucleation temperature). Only in this case evaporation occurs simultaneously throughout the liquid. In the entire liquid volume, vapor develops and strongly expands, entraining the remaining liquid. During this process, the liquid atomizes into small droplets, which allows an efficient further phase change. According to Reid [4,5], only such an evaporation process can be fast enough to produce significant blast. As a rule of thumb, Reid $[4,5]$ found the superheat limit temperature to be approximately equal to $90 \%$ of the critical temperature.

Although the superheat limit theory of explosive evaporation is widely accepted in the literature on explosions [1,2], it has not been directly proven to be true, due to the impossibility to do detailed measurements in the superheated liquid during a realistic BLEVE experiment. In fact, Birk et al. [6] have expressed doubt whether evaporation of superheated liquids can occur sufficiently fast to produce significant blast at all.

Direct measurements of the bubble growth process at the superheat limit were made using high speed camera observations of single small bubbles in a small amount of liquid [7-10]. It has become clear that vaporization close to the superheat limit is fast, because of not only the ease of bubble nuclei formation but also the properties of the surface of the growing bubbles. In [7], it is reported that the liquid-vapor interface in a vapor explosion process has a large-amplitude small-scale roughening during most of the evaporative stage in contrast to the smooth bubbles in conventional boiling. To make progress on the understanding and quantification of the blast generated by explosive evaporation, a well-designed experiment at a larger scale is needed. This experiment should show whether or not rapid evaporation is able to produce a significant blast, and give an indication of the conservatism in the current modeling.

The experiment described in this article concerns the BLEVE generated after a sudden rupture of a bottle filled with $\mathrm{CO}_{2}$ at high pressure. It has the advantage that the conditions in the vessel at the moment of rupture are well defined, and is such that the superheat limit, in principle, can be reached by depressurization. In the experiment, the rupture process is nearly instantaneous and complete, and the depressurization is not limited by a remaining containment. Therefore, this experiment can be used to validate models coupling submodels for the phase change and blast generation.

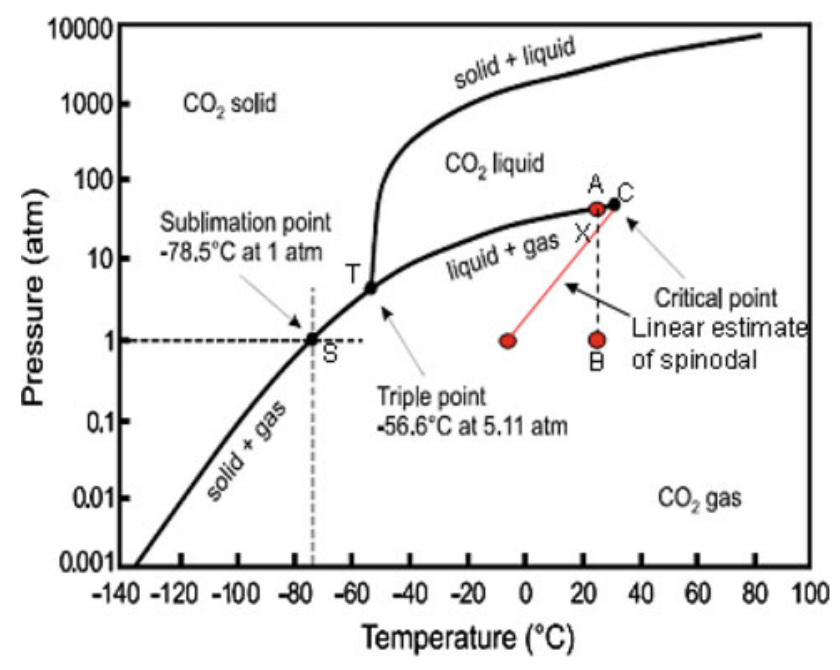

Fig. $1 P-T$ diagram of $\mathrm{CO}_{2}$ (adapted from Shakashiri [31])

In the rest of this introduction we first describe some of the complexity of the rapid evaporation of a superheated liquid and then introduce the simple model that will be applied in this article.

A distinction must be made between the thermodynamic superheat limit (TSL) and the kinetic superheat limit (KSL). The TSL temperature is a material property defined theoretically by the vanishing of the partial derivative at constant temperature of pressure to volume:

$\left(\frac{\partial p}{\partial v}\right)_{\mid T}=0$.

Equation (1) holds on a region in phase space called the spinodal. In Fig. 1, the approximate position of the liquid spinodal line in the $P T$ diagram of $\mathrm{CO}_{2}$ is represented as a straight line connecting the critical point $C$ to the experimentally determined superheat limit temperature at ambient pressure [2]. The depressurization at constant temperature would correspond to the line from $A$ to $B$. This line crosses the spinodal at the point indicated as $X$. Upon depressurization the system then will not reach $B$, but split up in a liquid and vapor phase, with the liquid phase remaining between the saturation curve and the spinodal. The TSL can be computed once an equation of state (EOS) describing the material is known. Different choices for the EOS can lead to different values [1,2]. De Sá et al. [11], Delale et al. [12] and Abbasi and Abbasi [2] have investigated whether or not the TSL derived from an EOS is in agreement with experiments. In particular, the study of Abbasi and Abbasi gives a comprehensive set of results.

The TSL predicts where the metastable liquid becomes intrinsically unstable, but it does not describe the kinetics of the evaporation process. On the other hand, the KSL is derived from kinetic theory formulae for the work of formation of critical bubbles, and is a model for phase change. The 
critical bubble size separates the cases of bubbles that will grow or will collapse. The KSL is the location in thermodynamic phase space where the critical bubble size becomes very small, and limits to the formation of new bubbles cease to exist. It contains as important quantities critical bubble size and critical bubble nucleation rate. To calibrate its prediction with experiments, KSL theory uses a presumed critical bubble nucleation rate [13]. For a properly chosen EOS, the experimentally determined KSL is quite close to the TSL.

To construct a complete theoretical model for rapid phase change of a superheated liquid, detailed submodels are needed for nucleation [14], bubble growth [10,15], bubblebubble interaction [16] and more generally the time evolution of the area of the interface between vapor and liquid. Pinhasi et al. [17] have formulated such a model. Their flow model consists of a combination of the Euler equations for the surrounding air and a two-phase model for the expanding liquid-vapor mixture. The two-phase model calculates at every position and time in the computational domain the rate of the evaporation. The model for evaporation distinguishes two regimes, one transport limited and one at the superheat limit described by expressions taken from kinetic theory. It is assumed that the liquid and the vapor have the same velocity and pressure, but a different temperature. Pinhasi et al. [17] report that the predictions of a $1 \mathrm{D}$ implementation of the model are in agreement with the calculation of blast effects using an energy estimate assuming irreversible expansion. But the model has not been directly validated with experiments. Recently, an application of the model to 2D cylindrically symmetric or 3D spherically symmetric situations has been made [18]. But applications in general geometries have not been reported yet.

In this article, we follow a simpler approach, still based on solution of the Euler equations. Two key aspects are taken into account in the model: the heat balance (first law of thermodynamics) and the momentum balance (Euler equation). It should first be pointed out that the rapid evaporation of the liquid, in general, does not lead to complete evaporation. Indeed, the heat of evaporation is extracted from the liquid, causing a decrease of the temperature and also of the vapor pressure of the liquid. The evaporation then stops when the liquid temperature has fallen to the boiling temperature at ambient pressure and consequently the vapor pressure has fallen to ambient pressure. From a calculated amount of evaporated mass, the involved amount of energy can then be calculated. The CCPS [19] and PGS 2 [20], for instance, recommend a TNT-equivalency based on the full quantity of work done by the vapor pressure-driven expansion over the entire temperature fall. van den Berg et al. [21,22] and van den Berg [23] go a step further and explicitly compute the gas dynamics of an explosive evaporation process numerically, starting from the assumption that the expansion is vapor pressure-driven and there are no limitations to the evaporation rate. This means that thermodynamic equilibrium at the interface between liquid and vapor is assumed. As a result there are negligible limitations on the transfer of heat and mass to the surface. The evaporation rate and the consequent expansion process are then fully determined by the inertia of the developing vapor and the surrounding atmosphere being pushed away. The heat needed for the evaporation is extracted uniformly from the liquid. The flow model is a homogeneous two-phase flow model in which a single set of Euler equations is solved. The fact that the evaporation, in general, is incomplete is taken into account in the form of an effective density of the liquid/vapor mixture represented as a single phase. This inertia-limited approach is obviously safe and conservative in its starting points. van den Berg et al. [21-23] have applied it to the experiments of Giesbrecht et al. [24] and have indeed found all predicted overpressure to be above the experimental overpressures.

The rest of this article is organized as follows: in Sect. 2 we report the experiments. Three 40-1 bottles of liquid carbon dioxide have been ruptured and the consequent blasts have been recorded. Section 3 subsequently presents results of numerical simulations using the inertia-limited modeling approach and an analysis of the agreement with the experimental observations. In Sect. 4, the conclusions are given.

\section{Experiments}

\subsection{Experimental set-up}

Proper experimental observation of the blast potential of superheated liquids requires a minimization of obscuring factors.

- The rupture of a pressure vessel should be fast and complete. If the rupture is caused by prolonged heating this is not the case, and the release rate of the liquid from the vessel will partly determine the blast effects.

- The vapor space in the vessel should be minimized. A substantial vapor space will act as an additional blast source, obscuring the blast from the explosive evaporation.

- A subsequent chemical explosion (combustion) of the vapor with air should be absent, to be able to focus on the blast from evaporation only.

Proper experimental observation of the blast potential of superheated liquids requires, therefore, a near-instantaneous and complete rupture of the vessel, a negligible vapor space, and the material should be non-combustible. In earlier experiments by Birk et al. [6], Giesbrecht et al. [24] and Johnson et al. [25], not all of these requirements were met, and are therefore less applicable to the objectives of this paper. 
Fig. 2 Schematic representations of the positions of bottle, pressure gauges and camera
Fig. 3 Left 40-1 carbon dioxide bottle equipped with cutting charges on either side. Right Pressure transducer Endevco 8530B mounted in a skimmer plate
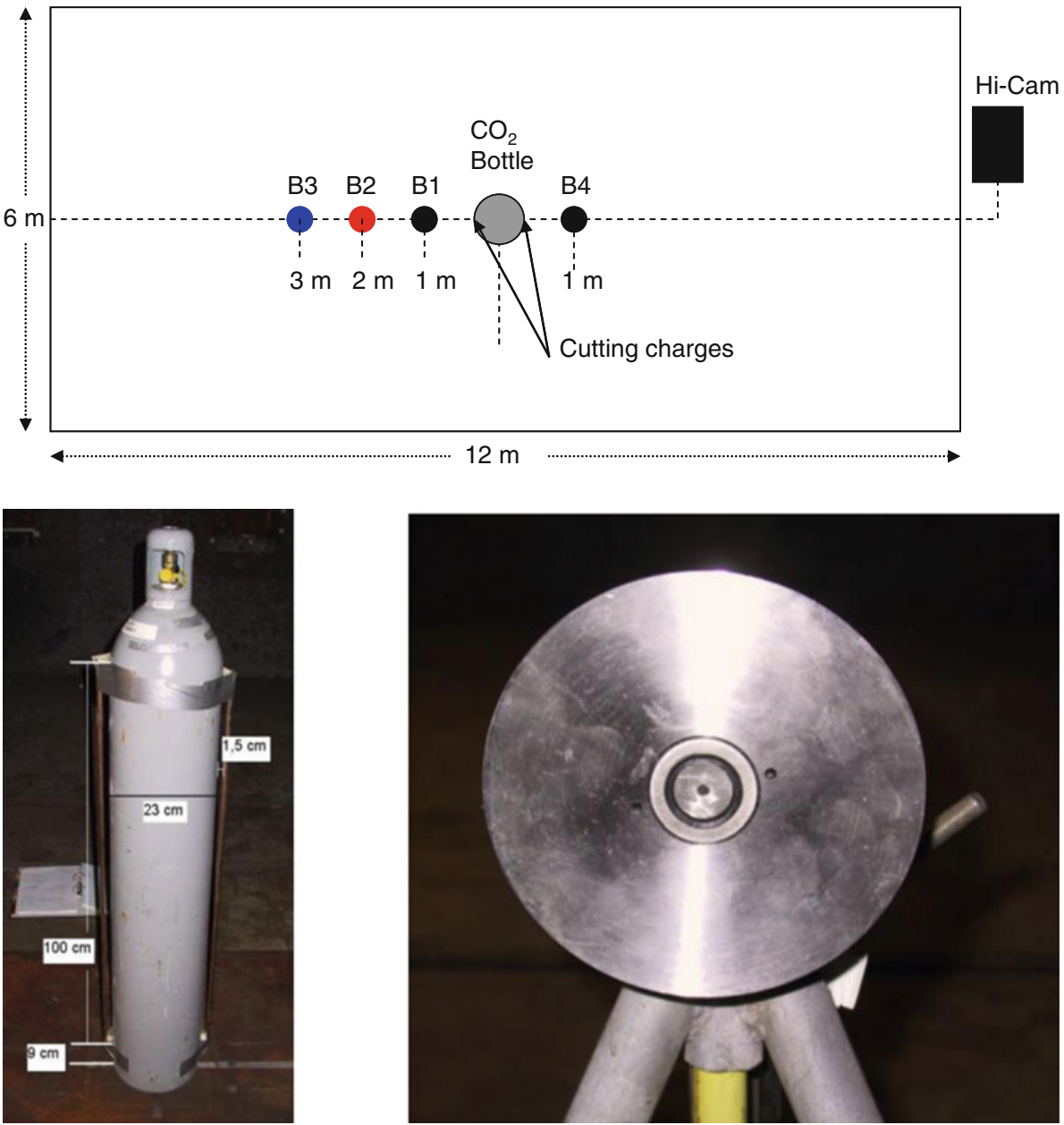

For the experiments described in this section, carbon dioxide $\left(\mathrm{CO}_{2}\right)$ was selected. The critical pressure and temperature of $\mathrm{CO}_{2}$ are $7.3 \mathrm{MPa}$ and $304 \mathrm{~K}$. Based on the information given in the previous section, the superheat limit temperature at ambient pressure can be estimated at $270 \mathrm{~K}$. This means that liquid $\mathrm{CO}_{2}$ stored under saturation pressure and at standard indoor laboratory temperatures (around $290 \mathrm{~K}$ ) is well above the superheat limit temperature. Furthermore, $\mathrm{CO}_{2}$ is readily available, and not combustible. Liquid $\mathrm{CO}_{2}$ is typically stored in heavy steel 401 cylindrical bottles. In our experiments, the bottles had a diameter of $0.23 \mathrm{~m}$, a height of $1.37 \mathrm{~m}$ and a wall thickness of about $8 \mathrm{~mm}$. The bottles were filled with liquid $\mathrm{CO}_{2}$ for more than $95 \%$.

The experiments have been performed at the Laboratory for Ballistic Research (TNO Defence, Security and Safety) in a test bunker with an internal volume of $6 \times 12 \times 4 \mathrm{~m}^{3}$. An overview of the experimental set-up is given in Fig. 2. The bottle was placed in a vertical position on the floor in the centre of the room (Fig. 3, left). In order to have a fast and complete rupture of the bottles, the rupture was initiated by cutting charges. Linear-shaped charges with a length of $1 \mathrm{~m}$ were installed at two opposite sides of the bottle. They were mounted at $1.5 \mathrm{~cm}$ distance from the bottle wall. The cutting charges consist of appropriately shaped copper tube filled with $90 \mathrm{~g} / \mathrm{m}$ RDX (detonation velocity $8,750 \mathrm{~m} / \mathrm{s}$ ). On detonation of the high-explosive, the shaped side of the tube produces a high-velocity jet of liquid copper that cuts through the steel wall of the bottle. Simultaneous detonation of the charges opened the bottles near-instantaneously over their full length at both sides. The aim was that in this way the cylindrical wall of the bottles ruptures in two halves.

To monitor the blast waves, pressure transducers (Endevco 8530B) were installed at several distances from the bottle in a plane defined by the axis of the bottle and the cutting charges. The pressure transducers were mounted in skimmer plates positioned flush to the anticipated flow (Fig. 3, right). Grease has been applied to the transducers to limit heat transfer during the experiment. Gauges B1, B2, B3, and B4 were positioned at $1,2,3$, and $1 \mathrm{~m}$ distance from the bottle, respectively, at a height of $0.7 \mathrm{~m}$ above the floor.

The pressure signals have been obtained using the SCADAS III system with a sampling rate of $202 \mathrm{kHz}$. Before the experiments, the total measurement chain of transducers, cables, amplifier and ADC was calibrated. To do this, 
Fig. 4 High speed camera frames recorded during experiment 2

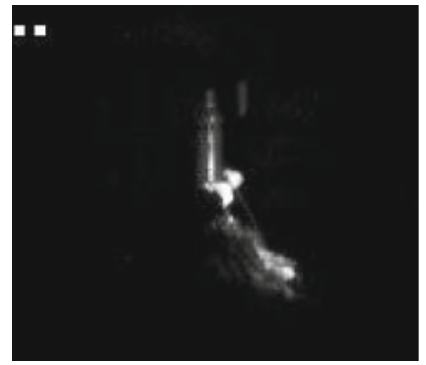

$-0.5 \mathrm{~ms}$

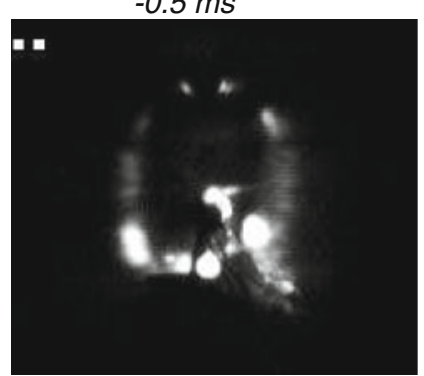

$1 \mathrm{~ms}$

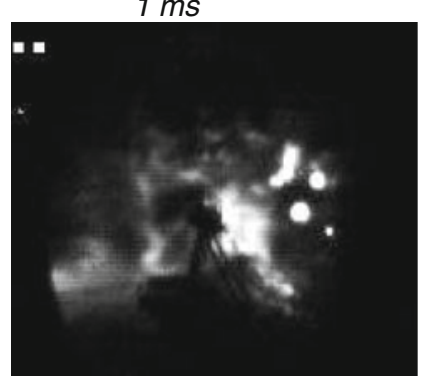

$2.5 \mathrm{~ms}$

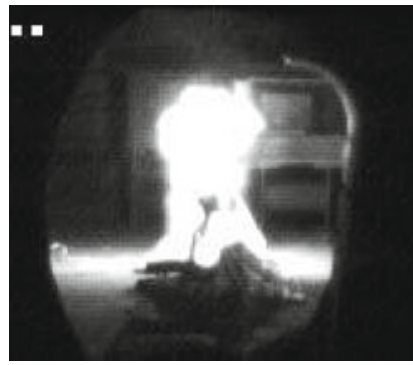

$0 \mathrm{~ms}$

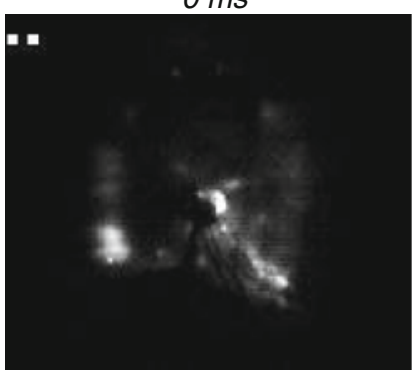

$1.5 \mathrm{~ms}$

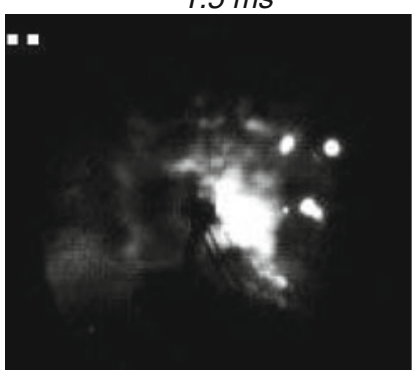

$3 \mathrm{~ms}$

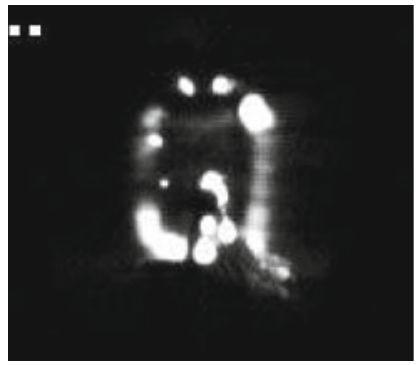

$0.5 \mathrm{~ms}$

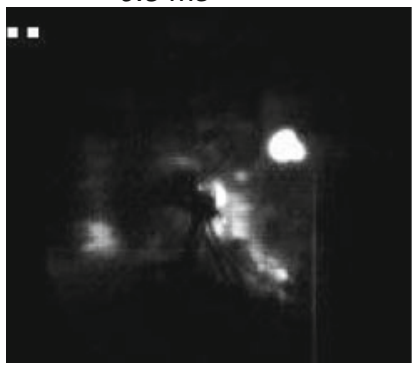

$2 m s$

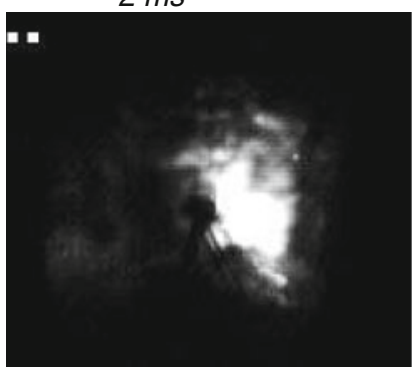

$3.5 \mathrm{~ms}$ the transducers were connected to a static pressure calibrator and loaded with different pressure values. With this calibration method the pressure can be measured with an accuracy of about $1 \%$. Because the opening of the bottles is not perfectly repeatable, stochastic variations were a dominant factor in the overall measurement error. The magnitude of this error could not be properly quantified due to the limited amount of performed experiments. Other sources of error are reflected blast waves from the walls and roof of the test bunker disturbing the events under interest. This phenomena starts to play a significant role after about $20 \mathrm{~ms}$. The measured pressure signals have been processed in the signal analysis program FAMOS. The colors used in Fig. 2 (black, red, and blue) correspond to the colors used for the pressure-time signals that are presented in the remainder of the article.

With this experimental set-up the large fragments of the bottle did not strike the pressure gauges. The fragmentation of the cutting charges and subsequent impacts at the equipment, however, formed a serious problem that could not be completely evaded. As a result the first experiment failed, while in experiments 2 and 3 not all of the pressure gauges worked properly. To eliminate the high frequency noise of the impacts, a low pass filter at $10 \mathrm{kHz}$ had to be applied.

\subsection{Experimental results}

\subsubsection{General observations}

Figure 4 shows a number of high speed camera frames recorded during the detonation of the cutting charges and subsequent evaporation in experiment 2 . The images provide a qualitative insight in the timing of the events, but unfortunately the blast wave cannot be clearly distinguished.

Figure 5 gives a typical example of the remains of the $\mathrm{CO}_{2}$ bottle. The bottle breaks up into two larger pieces and one or two smaller pieces (base plate and cap). In experiment 1 , only one small piece was recovered, namely the cap.

\subsubsection{Overpressure-time records}

Figures 6 and 7 show overpressure-time records observed at 1,2 and $3 \mathrm{~m}$ standoff in experiments 2 and 3, respectively.

All six records in Figs. 6 and 7 show an initial pulse at time zero, which is related to the triggering of the measurement equipment. The initial blast wave is caused by the cutting charges and its duration is about $1 \mathrm{~ms}$. It is followed by a blast wave of longer duration originating from the explosive 


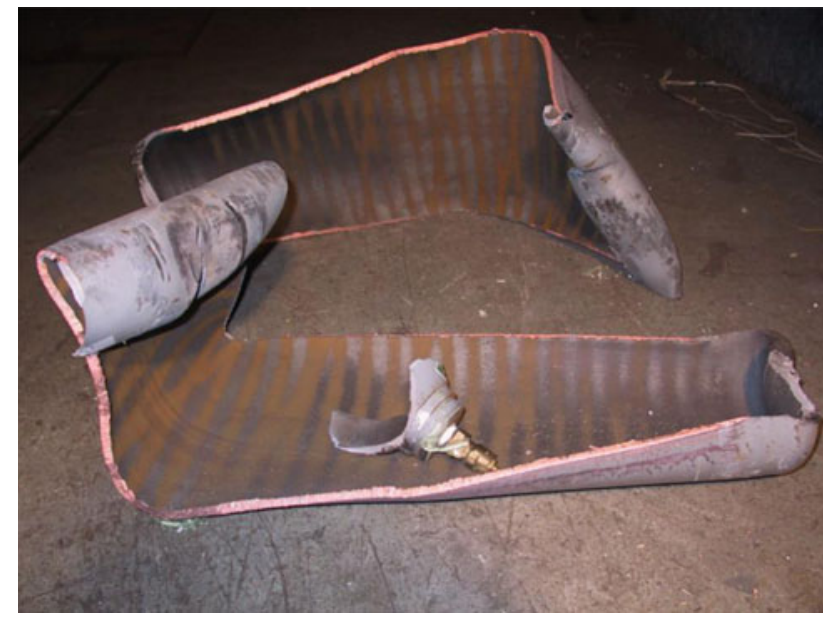

Fig. 5 Typical remains of the $\mathrm{CO}_{2}$ bottle (experiment 1 )

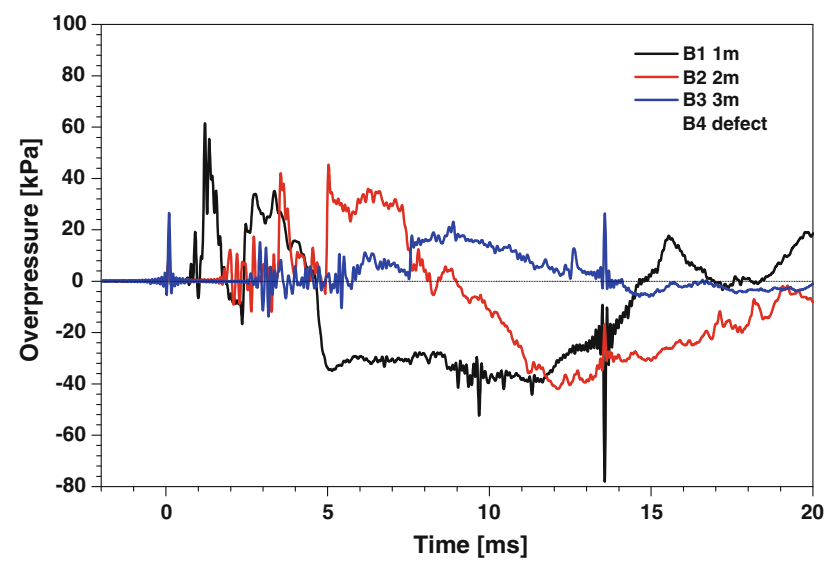

Fig. 6 Overpressure-time records observed at 1,2 and $3 \mathrm{~m}$ distance in experiment 2

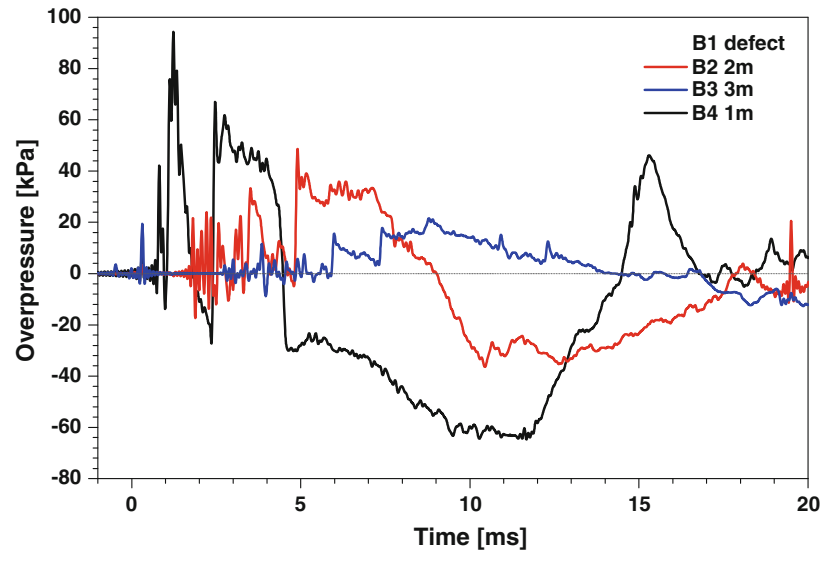

Fig. 7 Overpressure-time records observed at 1,2 and $3 \mathrm{~m}$ distance in experiment 3

evaporation process. Although initially higher in overpressure, the cutting charge blast is less energetic than the evaporation blast and has therefore a higher decay rate. With increasing distance, the evaporation blast is therefore gaining on the cutting charge blast and will overtake it in the far field.

The repeatability of the overpressure-time records in Figs. 6 and 7 is satisfactory. Deviation from perfect repeatability can be attributed to differences in energy absorbed by debris from the bottles and fragmentation of the cutting charges. We note that a fully controlled and symmetric opening of the bottles through the cutting charges is not certain and any asymmetries in the subsequent gas dynamics will be most prominent at short distance.

A characteristic development is observed in the overpressure-time signals recorded at $1 \mathrm{~m}$ distance from the bottles. This is a location inside the expanding carbon dioxide vapor. A positive overpressure phase of about $3 \mathrm{~ms}$ duration is followed by an abrupt fall to a long phase of substantial under pressure. The duration of the negative phase is about $10 \mathrm{~ms}$. The abrupt fall of the pressure indicates the passage of a shock that forms in the supersonic spatially diverging flow field of the expanding mixture of vapor and liquid carbon dioxide. Numerical modeling in Sect. 3 enables the simulation of these phenomena in detail and further analysis.

\section{Modeling}

\subsection{Introduction}

Physically realistic numerical modeling of the gas dynamic effects of an explosive evaporation event requires an advanced description of two-phase flow, modeling the mass, momentum and energy transfer between the vapor and the liquid phase for a void fraction that develops from $0 \%$ initially to near $100 \%$ in the end. Such an approach, which also considers the highly transient non-equilibrium thermodynamics of explosive evaporation, is still a big challenge.

A good first estimate of the strength of the resulting blast wave, however, can be obtained through a less sophisticated approach, using a single phase flow concept, described in van den Berg et al. [21,22] and van den Berg [23]. In this approach, the gas dynamics of the developing vapor in the ambient air is numerically modeled by a time step-wise integration of the Euler conservation equations for mass, momentum and energy. The Euler equations, using the Einstein summation convention, read:

$$
\begin{aligned}
& \frac{\partial \rho}{\partial t}+\frac{\partial}{\partial x_{j}}\left(u_{j} \rho\right)=0 \\
& \frac{\partial}{\partial t}\left(\rho u_{i}\right)+\frac{\partial}{\partial x_{j}}\left(u_{j} \rho u_{i}\right)+\frac{\partial p}{\partial x_{i}}=0 \\
& \frac{\partial}{\partial t}(\rho E)+\frac{\partial}{\partial x_{j}}\left(u_{j} \rho E\right)+\frac{\partial}{\partial x_{j}}\left(p u_{j}\right)=0,
\end{aligned}
$$


where $\rho$ is the density, $u_{i}$ the gas velocity in the $x_{i}$ direction, $p$ the pressure, $E$ the total specific energy, given by $E=e+1 / 2 u_{i} u_{i}, e$ the specific internal energy, given by $e=\frac{P}{\rho(\gamma-1)}, \gamma$ the ratio of specific heats, $x_{i=1,2,3}$ the coordinates in three spatial dimensions and $t$ is the time.

The basic concept of the modeling consists of a source area covering the initial liquid volume, from which a mix of vapor and liquid expands into the surroundings. The computational domain extends from the source boundary as far as necessary into the surroundings. The mass flow entering the computational domain via the boundary follows from the local conditions (pressure and density) in the source. The pressure prevailing at the boundary of the computational domain is determined from the assumption that the heat that has been extracted from the exploding system by the outflow of mixture has led to a simultaneous decrease of temperature and pressure in the liquid in the source area. The temperature decrease is determined from a heat balance and the pressure from the thermodynamic equilibrium condition (see Sect. 3.2).

The material that flows from the source into the computational domain behaves as a gas of high density. The density is higher than that of pure vapor, to take into account that in reality it is a mixture of vapor and atomized liquid. By assuming that the mixture can be treated as a gas, effects of surface tension and other two-phase effects are neglected (see Sect. 3.3). By letting the outflow velocities develop freely, the evaporation is always just as fast as the gas dynamics of expansion allows. Intrinsic thermodynamic limitations to the evaporation rate are not taken into account as factor that may possibly limit the strength of the explosion. The numerical method used for integration of the Euler equations is based on flux-corrected transport [26]. The model can be solved on a computational domain and numerical mesh of any dimensionality.

\subsection{Simple thermodynamics of explosive evaporation in the source area}

In the model of van den Berg et al. [21-23], evaporation is assumed to occur with the liquid and vapor at spatially uniform temperature and pressure, decreasing in time, following the saturation curve of the evaporating substance. For the application to $\mathrm{CO}_{2}$, the model of van den Berg et al. [21-23] has been generalized to the case of a system where the triple point $(T)$ is at higher than ambient conditions, the situation occurring in the $P-T$ diagram of $\mathrm{CO}_{2}$ (see Fig. 1). In this case the system is supposed to evolve from the initial state $A$ following the liquid-gas coexistence curve until the triple point $T$ and then following the solid-gas coexistence curve until the sublimation point $S$. While following this line the carbon dioxide evaporates (before $T$ ) or sublimates (after $T$ ). Possible mixing with surrounding air is not taken into
Table 1 Values of fitting constants

\begin{tabular}{lllll}
\hline Model parameters & $C_{A}$ & $C_{B}$ & $C_{C}$ & $\mathrm{~A}$ \\
\hline Unit & $\mathrm{kJ} \mathrm{kg}^{-1} \mathrm{~K}^{-1}$ & $\mathrm{~kJ} \mathrm{~kg}^{-1} \mathrm{~K}^{-1}$ & $\mathrm{~K}$ & - \\
Value & 1.85 & $4.81 \times 10^{-10}$ & 12.97 & 0.38 \\
\hline
\end{tabular}

account. To describe the thermodynamics of a carbon dioxide-air mixture a different $P-T$-diagram would be needed.

The evaporation/sublimation process requires heat, which is extracted from the remaining liquid/solid. The decrease of the liquid/solid mass and the fall of the liquid/solid temperature are related through:

$M_{1} C_{1} \mathrm{~d} T=L \mathrm{~d} M_{1}$,

where $M_{1}$ is the liquid/solid mass $(\mathrm{kg}), C_{1}$ the specific heat of the liquid/solid $\left(\mathrm{J} \mathrm{kg}^{-1} \mathrm{~K}^{-1}\right), L$ the heat of evaporation or sublimation $\left(\mathrm{J} \mathrm{kg}^{-1}\right), \mathrm{d} T$ the liquid/solid temperature change $(\mathrm{K})$ and $\mathrm{d} M_{1}$ is the liquid/solid mass change $(\mathrm{kg})$.

Both the specific heat of the liquid/solid $\left(C_{1}\right)$ and the heat of evaporation/sublimation (L) are dependent on the temperature, which has been approximated through curve fitting by the following relations $[20,27]$ :

$C_{1}=C_{A}+C_{B} \operatorname{EXP}\left[\frac{T}{C_{C}}\right]$
$L(T)=L\left(T_{0}\right) \cdot\left[\frac{T_{\mathrm{c}}-T}{T_{\mathrm{c}}-T_{0}}\right]^{A}$,

where $C_{1}$ is the specific heat $\left(\mathrm{kJ} \mathrm{kg}^{-1} \mathrm{~K}^{-1}\right), T$ the temperature $(\mathrm{K}), T_{0}$ the reference temperature, having the value $195 \mathrm{~K}$, $T_{\mathrm{c}}$ the critical temperature, having the value $305 \mathrm{~K}, L\left(T_{0}\right)$ the heat of sublimation at $T_{0}$, having the value $571 \mathrm{~kJ} \mathrm{~kg}^{-1}$, $C_{A}, C_{B}, C_{C}$ and $A$ are fitting constants (Table 1).

\subsection{Coupling of source area and gas dynamics}

The important transient properties in the source area, used as boundary conditions for the gas dynamics in the computational domain, are the temperature, the corresponding vapor pressure and the density of the vapor. The value of these quantities follows from the heat balance over the total source area. The vapor release rate (from evaporation or sublimation) is fully determined by the rate at which the developing vapor can expand in the surrounding air. The gas dynamics is computed by the (numerical) integration in time of the Euler equations under the continuously changing boundary conditions in the source.

The mass fraction that evaporates/sublimates (the flash fraction $F$ ) follows from the integration of relation (3) over the temperature drop, $\Delta T$, between the initial and a final liquid temperature. The specific heat and the heat of evaporation/sublimation of the remaining liquid/solid both are 
temperature-dependent along the trajectory. When the initial temperature is $290 \mathrm{~K}$ and the final temperature is the sublimation temperature $(195 \mathrm{~K})$, the value of the flash fraction can be obtained by numerical integration, and approximately equals $40 \%$.

Entrainment of atomized liquid/solid will of course slow the expansion process. In the present single-phase approach the entrainment has been accounted for by setting the density at the inflow boundary equal to the density of a mixture of vapor and entrained liquid. In the literature this is also known as a homogeneous flow model for a two-phase mixture. The density of the two-phase mixture after the flash fraction has evaporated is approximated equal to the vapor density divided by the flash fraction $F$. Inside the computational domain the fluid is considered a compressible ideal gas.

\subsection{Simulation of the experiment}

The model representation of the initial state of the experiment consists of a source area covering the initial liquid volume and a surrounding with stagnant air. The conditions (pressure and temperature) inside the source area are computed using global thermodynamic equilibrium as described above. The computational domain consists of the region outside the source area and inflow boundary conditions are specified at the edge of the source area.

We start with the further simplification to a one-dimensional spherical symmetry in Sect. 3.4.1. This simulation is less representative for the actual experimental configuration of course, but facilitates detailed analysis of the observed spatial and temporal flow field characteristics. A more realistic simulation of the experiment is presented in Sect. 3.4.2, where we present results for cylinder symmetry. The influence of the launched bottle halves on the gas dynamics can however not be considered.

\subsubsection{Simulation assuming spherical symmetry}

The explosive evaporation of 40 liters of carbon dioxide in the experimental setup described above has been modeled through the specification of a hemispherical source area of $0.267 \mathrm{~m}$ radius on a horizontal surface in a one-dimensional mesh of spherical symmetry, i.e. with radius $r$ as only independent spatial coordinate. The resolution was chosen as 50 nodes per source area radius, i.e. a cell size of $5.3 \mathrm{~mm}$. The initial temperature and pressure of the liquid $\mathrm{CO}_{2}$ was $290 \mathrm{~K}$ and 5.2 MPa. The transient evolution of the overpressure at 1,2 and $3 \mathrm{~m}$ distance from the origin has been recorded. The records are represented in Fig. 8.

Comparing the computed overpressure-time records with the experimentally observed ones (Figs. 6,7), we note that this one-dimensional computation leads to an overestima-

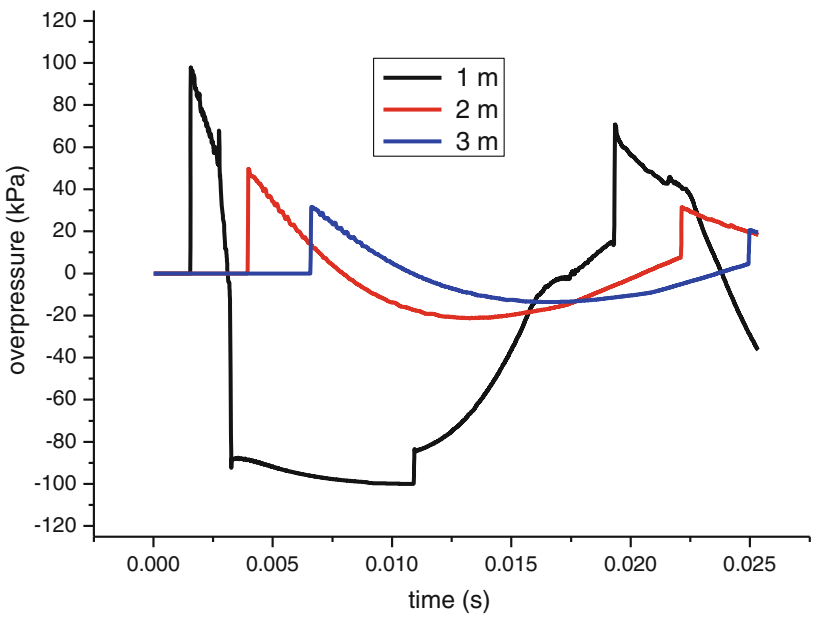

Fig. 8 Overpressure-time developments recorded at 1,2 and $3 \mathrm{~m}$ distance calculated in a one-dimensional mesh of spherical symmetry around a source area initially containing $40-1$ liquid $\mathrm{CO}_{2}$ at $290 \mathrm{~K}$

tion of the overpressures in terms of both the positive and the negative phases. This may not be very surprising because all starting points in the physical modeling have been safe and conservative. The overpressure-time records at $1 \mathrm{~m}$ show a striking qualitative resemblance. A positive overpressure phase is followed by an abrupt fall to a long phase of substantial under pressure as described in Sect. 2.2.

This phenomenon is very characteristic for the near field gas dynamics of any explosion of large expansion ratio and can be further studied by analyzing the plots of the flow parameter distributions at a few consecutive points in time in Fig. 9. Figure 9 represents full density (black), overpressure (red) and velocity fields (blue) due to the explosive evaporation of 401 of carbon dioxide at a few consecutive points in time. Both density and overpressure have been non-dimensionalized with the density and pressure of ambient air. The source area has been indicated and extends up to a radius of $0.267 \mathrm{~m}$. The vapor conditions in terms of vapor pressure and density in the source area are the boundary conditions for the gas dynamics in the region outside the source area. The mixture of liquid and gaseous $\mathrm{CO}_{2}$ that starts flowing out of the source area pushes aside and thereby compresses the ambient air like an expanding piston. The air is compressed in a shock that precedes the flow field. Outside the flow field the ambient air is quiescent.

Successively from right to left in all of the distributions in Fig. 9, the following phenomena are visible:

- A lead shock of the flow field that compresses the ambient air.

- A contact surface between the compressed ambient air and the mixture of gaseous and liquid carbon dioxide, which is characterized by a substantial density jump. The density of the carbon dioxide mixture at the contact 

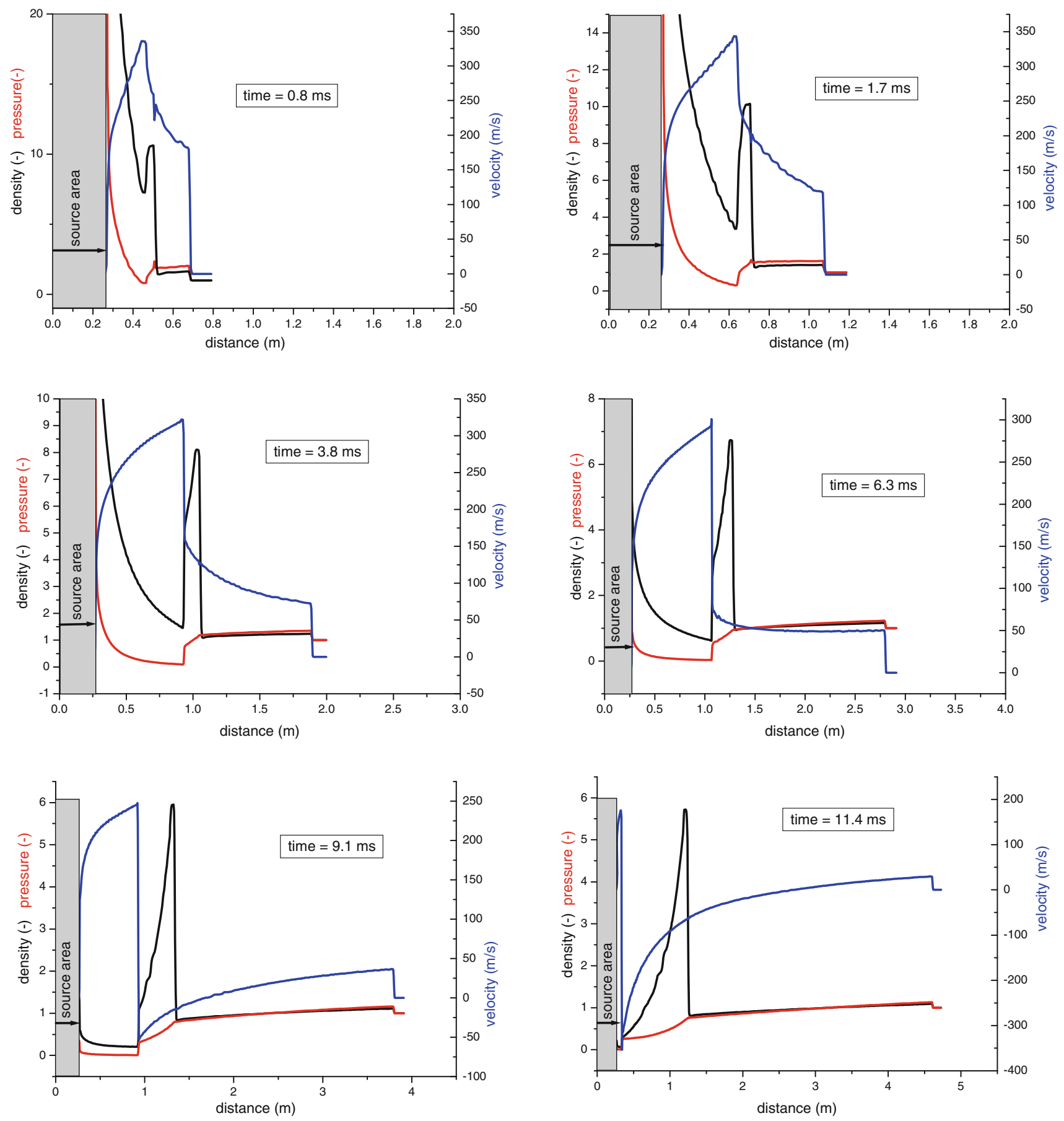

Fig. 9 Density, pressure and velocity fields at a few consecutive points in time, calculated in a spherical mesh around a source area initially containing 401 liquid $\mathrm{CO}_{2}$ at $290 \mathrm{~K}$

surface is quite high because it contains atomized liquid and it has expanded from the initial vapor pressure of 5.2 MPa down to almost ambient.

- A shock, which spontaneously forms just inside the expanding bubble of carbon dioxide. This shock is the consequence of the supersonic velocities that develop in the spatially expanding outflow of carbon dioxide from the source. In the spatially diverging supersonic flow field pressure fall goes hand in hand with velocity increase. The shock recompresses the flow to subsonic, which provides compatibility with the flow behind the decaying lead shock. Initially the shock inside the carbon dioxide bubble is driven outward by the swelling outflow. Within the shock radius, the carbon dioxide expands 
down to near-vacuum, while flow velocities decrease. Lower velocities in the flow field allow the shock to propagate upstream back to the origin where it focuses and subsequently reflects. Focusing and reflection go hand in hand with recompression and secondary expansion, which induces a secondary wave propagating after the primary.

All of the here simulated phenomena are found back in the experimentally observed overpressure records at $1 \mathrm{~m}$ distance in the Figs. 6 and 7. The passage of the lead shock that compresses the ambient air is observed at about $2 \mathrm{~ms}$ followed by the passage of the shock that formed inside the expanding $\mathrm{CO}_{2}$ bubble at about $5 \mathrm{~ms}$. Behind this shock the bubble expands down to almost vacuum. The experimental observation of the passage of the same shock subsequently propagating backward to the origin is less clear. Finally, the secondary blast wave appears, which may be of substantial strength.

\subsubsection{Simulation assuming cylindrical symmetry}

The explosive evaporation of 401 of $\mathrm{CO}_{2}$ in the experimental set-up has also been modeled through the specification of a vertical cylindrical source area of $11 \mathrm{~cm}$ radius and $110 \mathrm{~cm}$ height on the earth's surface. The volume of the source area and the surrounding space was meshed using a two-dimensional mesh of cylindrical symmetry of $400 \times 800$ cells of $1 \times 1 \mathrm{~cm}^{2}$ cell size.

Some snapshots of the development of the pressure distribution around the evaporating $\mathrm{CO}_{2}$ are represented in Fig. 10. The left side of the pictures is the cylinder axis of the mesh, which is positioned through the centre line of the source area. Figure 10 shows the pressure distributions using color shading and a set of isobar lines. Colors correspond to pressure levels through a color bar. Where isobar lines accumulate, shock phenomena are visible. The pictures show how a blast flow field expands preceded by a shock. Around the source, an area of substantial under pressure develops. The overpressure in the blast wave and the under pressure around the source are connected in a shock. This shock propagates up the spatially diverging supersonic expansion flow of $\mathrm{CO}_{2}$ from the source. Because initially the swelling flow velocity is higher than the propagation velocity of the shock, the shock is driven outward. When the outflow velocities decrease because the source runs out of $\mathrm{CO}_{2}$, the shock is able to move upstream back to the source where it focuses and reflects and becomes the origin of a secondary blast wave running after the primary.

The evolution of overpressure has been recorded at 1,2 and $3 \mathrm{~m}$ distance from the bottle axis at a vertical position of $0.7 \mathrm{~m}$ above the earth's surface. The records are presented in Fig. 11.
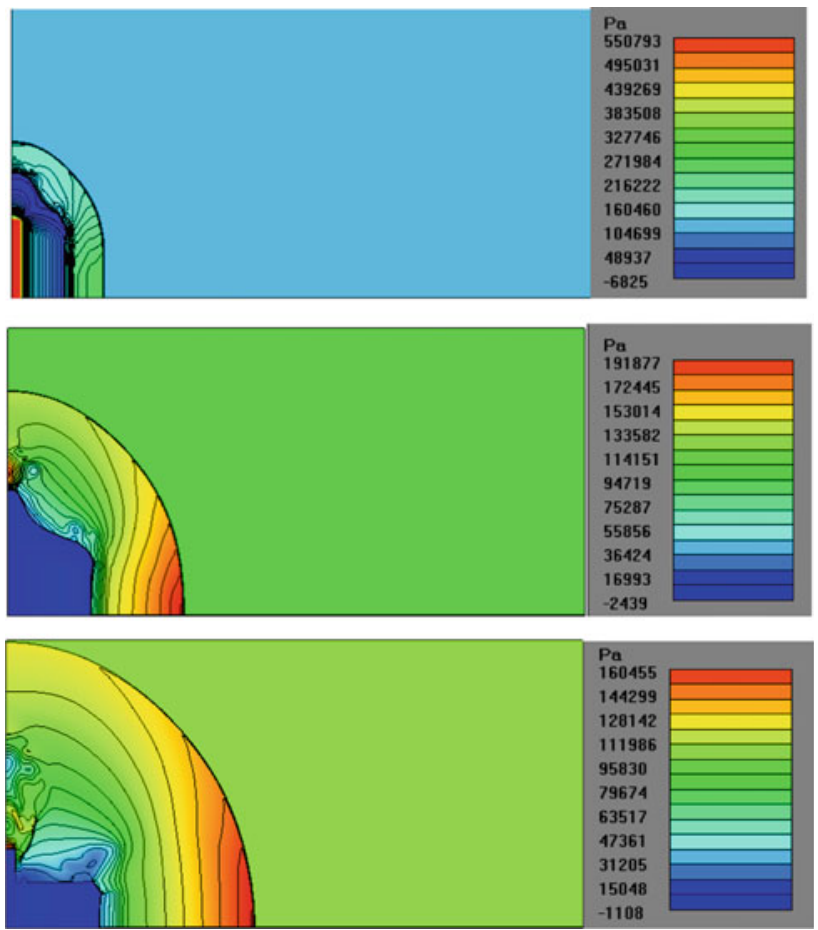

Fig. 10 Pressure distributions produced by the explosive evaporation of 401 of liquid $\mathrm{CO}_{2}$ at a temperature of $290 \mathrm{~K}$

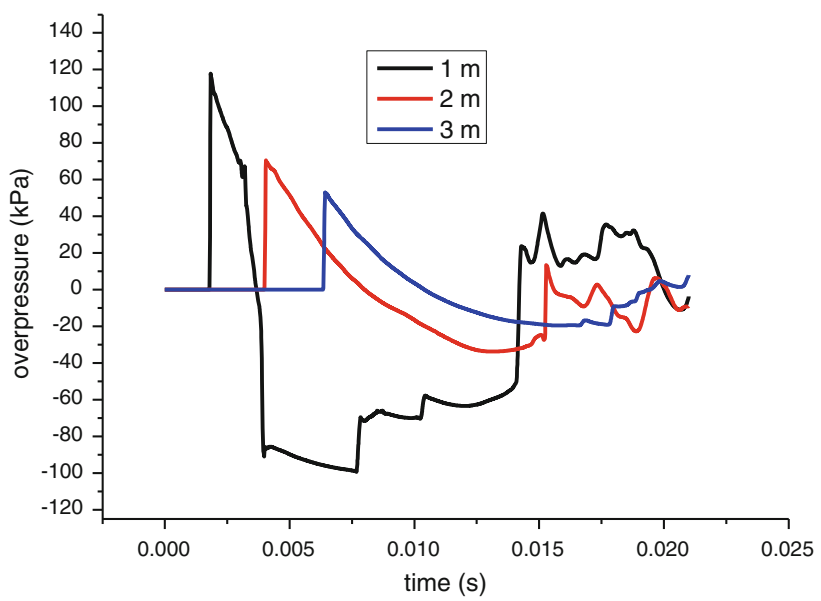

Fig. 11 Overpressure-time developments recorded at 1,2 and $3 \mathrm{~m}$ distance from the axis of the cylinder and at height $0.7 \mathrm{~m}$, calculated in a two-dimensional mesh of cylindrical symmetry around a source area initially containing 401 liquid $\mathrm{CO}_{2}$ at $290 \mathrm{~K}$

The records computed in the two-dimensional cylindrical mesh show a development only slightly different from the records compiled in the one-dimensional spherical mesh. The blast overpressures are now higher because at short distances from the cylindrical source the spatial expansion of the gas dynamics is fully two-dimensional. The computed blast peak overpressures are roughly a factor $1.5-2$ higher than experimentally observed. The duration of the overpressure is slightly too short. The duration of the negative phase of the record observed at $1 \mathrm{~m}$ distance is more or less reproduced. 


\subsubsection{Assessment}

So far, the modeling of the gas dynamics of an explosive evaporation process in this paper has been conservative in all starting points and assumptions. By letting the outflow velocities from the source area develop freely, the implicit assumption was that there are no intrinsic thermodynamic limitations to the evaporation rate. The evaporation rate becomes then as fast as the inertia in the expansion process of carbon dioxide into the surrounding atmosphere allows. Possible intrinsic thermodynamic limitations to the evaporation process are fully neglected in this way. If sufficient experimental data are available, the fact that the model does not take into account a possible limitation due to finite evaporation rate can be simply remedied by artificially reducing the outflow velocities from the source domain. In this way, the blast computed by the modeling can simply be made to fully correspond to the experimental observations. In the absence of sufficient experimental data, such tuning is impossible and applying the model as such in hazardous materials safety investigations or comprehensive industrial risk analyses could lead to an overestimation of blast effects and consequently to an overestimation of risk and thereby to undesirable higher cost. To avoid this, there is a need for more precise modeling approaches.

\section{Conclusions}

We have carried out well-defined experiments on the explosive evaporation of the contents of 40-1 bottles of liquid $\mathrm{CO}_{2}$. The measured blast wave can be used for the validation of BLEVE blast prediction models.

The inertia-limited modeling and simulation approach to BLEVE [21-23] has been validated by its application to these experiments. This has been done firstly in a spherically symmetric one-dimensional approximation and secondly in a two-dimensional cylindrically symmetric approximation. Good qualitative agreement between model and experiment was found, in particular concerning the strength and duration of overpressure and rarefaction waves. Quantitatively, the inertia-limited model provides a safe estimate of the BLEVE blast due to its conservative assumptions.

More realistic prediction of BLEVE blast is desirable in the field of risk assessment for the process industry. In order to clarify the limitations of the inertia-limited modeling approach, the physics of the rapid evaporation of superheated liquids has been reviewed. It is expected that further development of the approach pioneered by Pinhasi et al $[17,18]$ can potentially lead to a better quantitative agreement between modeling and experiment. This will make it possible to quantify the relative importance of the different physical phenomena that are not explicitly taken into account in the inertia-limited model, such as bubble nucleation and growth rate, and two-phase flow regime.
In order to apply the type of modeling proposed by Pinhasi et al. $[17,18]$ to the experiments described above, the following submodels have to be developed

- The thermodynamic model has to be elaborated. Care is to be taken that it is predicting not only the properties at equilibrium but also the properties in the metastable state (density, sound speed, etc.).

- The mass and heat transfer at fast evaporation is to be described accurately. Non-equilibrium thermodynamics [28] provides the most accurate formulation. Molecular dynamics simulations can be used to related interface properties to bulk phase properties of the metastable state [30]. In a first approach simple relaxation models, using a characteristic time scale for the transfer process can be used. The latter allows studying the dependence of the results on the assumed relaxation time, which is a measure for the rate of evaporation.

- The initial rapid decrease of pressure at first contact of the superheated liquid with the surrounding air has to be described in detail.

The Eulerian-Eulerian BLEVE model described above has been implemented and first results reported in reference (Xie and Roekaerts [30]). The results of the application to the experiment described in Sect. 2 will be reported elsewhere.

For future experimentation it is recommended to choose a free field test site instead of a test bunker, in order to exclude disturbances from blast wave reflections. This becomes especially relevant when experiments at a larger scale (i.e. bottle volume) are carried out. Another recommendation is to measure the blast in multiple directions to capture the directional dependence caused by the fact that the opening of the bottles is not perfectly repeatable. Besides experiments at a larger scale, also the dependency of blast on variations in temperature would be of interest for validation purposes.

Acknowledgments We gratefully acknowledge interesting discussions with P. Colonna (TU Delft), S. Kjelstrup and D. Bedeaux (TU Delft and NTNU, Trondheim), and A. Prosperetti (University Twente and John Hopkins University). The work of M. Xie is financially supported by Delft Cluster as part of the project "Innovatief Ondiep Bouwen".

Open Access This article is distributed under the terms of the Creative Commons Attribution License which permits any use, distribution, and reproduction in any medium, provided the original author(s) and the source are credited.

\section{References}

1. Abbasi, T., Abbasi, S.A.: The boiling liquid expanding vapor explosion (BLEVE): mechanism, consequence assessment, management. J. Hazard. Mater. 141, 489-519 (2007)

2. Abbasi, T., Abbasi, S.A.: Accidental risk of superheated liquids and a framework for predicting the superheat limit. J Loss Prev. Process Ind. 20, 165-181 (2007) 
3. Casal, J., Salla, J.M.: Using liquid superheating energy for a quick estimation of overpressure in BLEVEs and similar explosions. J. Hazard. Mater. A 137, 1321-1327 (2006)

4. Reid, R.C.: Superheated liquids. Am. Sci. 64, 146-156 (1976)

5. Reid, R.C.: Possible mechanisms for pressurized-liquid tank explosions or BLEVE's. Science 203, 3 (1979)

6. Birk, A.M., Davison, C., Cunningham, M.: Blast overpressures from medium scale BLEVE tests. J. Loss Prev. Process Ind. 20(3), 194-206 (2007)

7. Shepherd, J.E., Sturtevant, B.: Rapid evaporation at the superheat limit. J. Fluid. Mech. 121, 379-402 (1982)

8. Frost, D.L., Sturtevant, B.: Effects of ambient pressure on the instability of a liquid explosively boiling at the superheating limit. J. Heat Transf. 108, 418-424 (1986)

9. McCann, H., Clarke, L.J., Masters, A.P.: An experimental study of vapor growth at the superheating limit temperature. Int. J. Heat Mass Transf. 32, 1077-1093 (1989)

10. Lesin, S., Baron, A., Branover, H., Merchuk, J.C.: Experimental studies of direct contact boiling at the superheat limit. High Temp. 31(6), 866-884 (1993)

11. De Sá, E.M., Meyer, E., Soares, V.: Adiabatic nucleation in the liquid-vapor phase transition. J. Chem. Phys. 114(19), 8505-8510 (2001)

12. Delale, C.F., Hruby, J., Marsik, F.: Homogeneous bubble nucleation in liquids: the classical theory revisited. J. Chem. Phys. 118(2), 792-806 (2003)

13. Blander, M., Katz, J.L.: Bubble nucleation in liquids. AIChE J. 21, 833-848 (1975)

14. Riznic, J.A., Ishii, M.: Bubble number density and vapor generation in flashing flow. Int. J. Heat Mass Transf. 32(10), 1821-1833 (1989)

15. Sher, E., Bar-Kohany, T., Rashkovan, A.: Flash boiling atomization. Prog. Energy Combust. Sci. 34, 417-439 (2008)

16. Deligiannis, P., Cleaver, J.W.: Influence of surrounding bubbles on the rate of nucleation. Int. J. Heat Mass Transf. 36(15), 36973701 (1993)

17. Pinhasi, G.A., Ullmann, A., Dayan, A.: 1D plane numerical model for boiling liquid expanding vapor explosion. Int. J. Heat Mass Transf. 50, 4780-4795 (2007)

18. Pinhasi, G.A., Dahan, Y., Dayan, A., Ullmann, A.: Modeling of boiling liquid expanding vapor explosion (BLEVE): plane, cylindrical and spherical 1D model. In: Proceedings of the 9th Biennial ASME Conference on Engineering Systems Design and Analysis, ESDA08, Haifa, Israel, ESDA2008-5933, July 7-9 (2008)
19. CCPS: Guidelines for the evaluation of the characteristics of vapor cloud explosions, flash fires and BLEVES. Center for Chemical Process Safety, AIChE (1994)

20. PGS 2 (Yellow Book): Methods for the calculation of physical effects, Publicatiereeks Gevaarlijke Stoffen 2 (2005). http://www. publicatiereeksgevaarlijkestoffen.nl/publicaties/PGS2.html

21. van den Berg, A.C., van der Voort, M.M., Weerheijm, J., Versloot, N.H.A.: Expansion-controlled evaporation-a safe approach to BLEVE blast. J. Loss Prev. Process Ind. 17, 397-405 (2004). doi:10.1016/j.jlp.2004.07.002

22. van den Berg, A.C., van der Voort, M.M., Weerheijm, J., Versloot, N.H.A.: BLEVE blast by expansion controlled evaporation. Process Saf. Prog. 25(1), 44-51 (2006). doi:10.1002/prs.10116

23. van den Berg, A.C.: Blast charts for explosive evaporation of superheated liquids. Process Saf. Prog. 27(3), 219-224 (2008). doi:10. 1002/prs. 10252

24. Giesbrecht, H., Hess, K., Leuckel, W., Maurer, B.: Analysis of explosion hazards on spontaneous release of inflammable gases in the atmosphere. Part 1: Propagation and deflagration of vapor clouds on the basis of bursting tests on model vessels. Ger. Chem. Eng. 4, 305-314 (1981)

25. Johnson, D.M., Pritchard, M.J.: Large-scale experimental study of boiling liquid expanding vapor explosions (BLEVEs). In: The 14th International LNG/LPG Conference and Exhibition, Amsterdam, 4-7 December (1991)

26. Boris, J.P.: Flux-corrected transport modules for solving generalized continuity equation NRL memorandum. Report no.3237, Naval Research Laboratory, Washington (DC) (1976)

27. Engineering Toolbox. http://www.engineeringtoolbox.com/carbon -dioxide-d_1000.html (2009, accessed 2010)

28. Kjelstrup, S., Bedeaux, D.: Non-Equilibrium Thermodynamics of Heterogeneous Systems. World Scientific, New Jersey (2008)

29. Kraska, T., Roemer, F., Imre, A.R.: The relation of interface properties and bulk phase stability: molecular dynamics simulations of carbon dioxide. J. Phys. Chem. B 113(14), 4688-4697 (2009)

30. Xie, M., Roekaerts, D.: Thermodynamic and gas dynamic aspects of a boiling liquid expanding vapor explosion, Dutch Process Technology Symposium (NPS2009), Veldhoven (2009)

31. Shakashiri: http://www.scifun.org (2008, accessed 2009) 